\title{
@creative
commons
}

\section{Partial product of graphs and Vizing's conjecture}

\author{
Ismael González Yero \\ Departamento de Matemáticas, Escuela Politécnica Superior de Algeciras \\ Universidad de Cádiz, Av. Ramón Puyol s/n, 11202 Algeciras, Spain
}

Received 9 December 2012, accepted 19 December 2013, published online 5 June 2014

\begin{abstract}
Let $G$ and $H$ be two graphs with vertex sets $V_{1}=\left\{u_{1}, \ldots, u_{n_{1}}\right\}$ and $V_{2}=\left\{v_{1}, \ldots, v_{n_{2}}\right\}$, respectively. If $S \subset V_{2}$, then the partial Cartesian product of $G$ and $H$ with respect to $S$ is the graph $G \square_{S} H=(V, E)$, where $V=V_{1} \times V_{2}$ and two vertices $\left(u_{i}, v_{j}\right)$ and $\left(u_{k}, v_{l}\right)$ are adjacent in $G \square_{S} H$ if and only if either $\left(u_{i}=u_{k}\right.$ and $\left.v_{j} \sim v_{l}\right)$ or $\left(u_{i} \sim u_{k}\right.$ and $v_{j}=v_{l} \in S$ ). If $A \subset V_{1}$ and $B \subset V_{2}$, then the restricted partial strong product of $G$ and $H$ with respect to $A$ and $B$ is the graph $G_{A} \otimes_{B} H=(V, E)$, where $V=V_{1} \times V_{2}$ and two vertices $\left(u_{i}, v_{j}\right)$ and $\left(u_{k}, v_{l}\right)$ are adjacent in $G_{A} \otimes_{B} H$ if and only if either $\left(u_{i}=u_{k}\right.$ and $\left.v_{j} \sim v_{l}\right)$ or $\left(u_{i} \sim u_{k}\right.$ and $\left.v_{j}=v_{l}\right)$ or $\left(u_{i} \in A, u_{k} \notin A, v_{j} \in B, v_{l} \notin B, u_{i} \sim u_{k}\right.$ and $\left.v_{j} \sim v_{l}\right)$ or $\left(u_{i} \notin A, u_{k} \in A, v_{j} \notin B, v_{l} \in B, u_{i} \sim u_{k}\right.$ and $\left.v_{j} \sim v_{l}\right)$. In this article we obtain Vizing-like results for the domination number and the independence domination number of the partial Cartesian product of graphs. Moreover we study the domination number of the restricted partial strong product of graphs.
\end{abstract}

Keywords: Domination, partial product of graphs, Cartesian product graph, strong product graph, Vizing's conjecture.

Math. Subj. Class.: 05C69, 05C70, 05C76

\section{Introduction}

Vizing's conjecture [7] is perhaps one of the most popular open problems related to domination in graphs. It states that the domination number of the Cartesian product of two graphs is at least as large as the product of their domination numbers. A high quantity of approaches to that problem have been developed in this sense. The surveys [1,3] are very complete compilations of the principal results obtained in this topic. Moreover, several Vizing-like results for some other kind of product graphs have been also published [5]. In

E-mail address: ismael.gonzalez@uca.es (Ismael González Yero) 
this article we introduce the notion of partial Cartesian product of graphs and obtain some corresponding Vizing-like results for the domination number and independence domination number of partial Cartesian product of graphs.

We establish first the principal terminology and notation which we will use throughout the article. Hereafter $G=(V, E)$ denotes a finite simple graph. The complement of a set $D$ is denoted by $\bar{D}$. A set $D$ is a dominating set if every vertex of $\bar{D}$ is adjacent to a vertex of $D$ [4]. A dominating set $D$ is called minimal if does not contain any dominating set as a proper subset. The domination number, $\gamma(G)$, is the minimum cardinality of any dominating set in $G$. We say that a set $S$ is a $\gamma(G)$-set if it is a dominating set and $|S|=\gamma(G)$. The following result, due to Ore, can be found in [4].

Lemma 1.1. [4] A dominating set $S$ is a minimal dominating set of a graph $G=(V, E)$ if and only if for each $u \in S$ one of the following conditions holds:

- $u$ is an isolated vertex of $S$,

- there exists $v \in V-S$ for which $N(v) \cap S=\{u\}$.

\section{Results}

Let $G$ and $H$ be two graphs with set of vertices $V_{1}=\left\{u_{1}, \ldots, u_{n_{1}}\right\}$ and $V_{2}=\left\{v_{1}, \ldots, v_{n_{2}}\right\}$, respectively. We recall that the Cartesian product of $G$ and $H$ is the graph $G \square H=(V, E)$, where $V=V_{1} \times V_{2}$ and two vertices $\left(u_{i}, v_{j}\right)$ and $\left(u_{k}, v_{l}\right)$ are adjacent in $G \square H$ if and only if one of the following conditions is satisfied:

- $u_{i}=u_{k}$ and $v_{j} \sim v_{l}$, or

- $u_{i} \sim u_{k}$ and $v_{j}=v_{l}$.

The strong product of $G$ and $H$ is the graph $G \otimes H=(V, E)$, where $V=V_{1} \times V_{2}$ and two vertices $\left(u_{i}, v_{j}\right)$ and $\left(u_{k}, v_{l}\right)$ are adjacent in $G \otimes H$ if and only if one of the following conditions is satisfied:

- $u_{i}=u_{k}$ and $v_{j} \sim v_{l}$, or

- $u_{i} \sim u_{k}$ and $v_{j}=v_{l}$, or

- $u_{i} \sim u_{k}$ and $v_{j} \sim v_{l}$.

For a vertex $u_{i} \in V_{1}$, the subgraph of $G \square H$ or $G \otimes H$ induced by the set $\left\{\left(u_{i}, v\right) \mid v \in\right.$ $\left.V_{2}\right\}$ is called an $H$-layer or an $H$-fiber. Similarly, for $v_{j} \in V_{2}$, the subgraph induced by $\left\{\left(u, v_{j}\right) \mid u \in V_{1}\right\}$ is a $G$-layer or a $G$-fiber.

\subsection{Partial Cartesian product of graphs}

Let $G$ and $H$ be two graphs with set of vertices $V_{1}=\left\{u_{1}, \ldots, u_{n_{2}}\right\}$ and $V_{2}=\left\{v_{1}, \ldots, v_{n_{1}}\right\}$, respectively. If $S \subset V_{2}$, then the partial Cartesian product of $G$ and $H$ with respect to $S$ is the graph $G \square_{S} H=(V, E)$, where $V=V_{1} \times V_{2}$ and two vertices $\left(u_{i}, v_{j}\right)$ and $\left(u_{k}, v_{l}\right)$ are adjacent in $G \square_{S} H$ if and only if one of the following conditions is satisfied:

- $u_{i}=u_{k}$ and $v_{j} \sim v_{l}$, or

- $u_{i} \sim u_{k}$ and $v_{j}=v_{l} \in S$. 


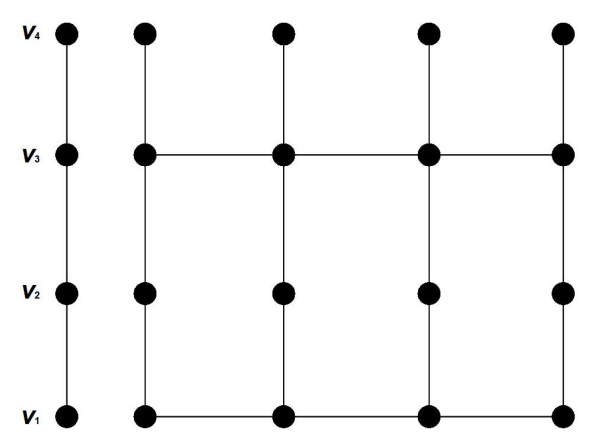

Figure 1: The partial Cartesian product graph $P_{4} \square_{\left\{v_{1}, v_{3}\right\}} P_{4}$.

An example of a partial Cartesian product graph is shown in Figure 1. Also notice that the partial Cartesian product of $G$ and $H$ is the spanning subgraph of $G \square H$ obtained by deleting the edges of $G \square H$ in the $H$-fibers that correspond to vertices from $V_{2} \backslash S$.

Notice that if $S=V_{2}$, then $G \square_{S} H$ is the standard Cartesian product graph $G \square H$. Also, note that if $S$ is formed by only one vertex $v$, then $G \square_{S} H$ is a particular case of the rooted product graph $G \circ_{v} H$ defined in [2]. Domination related parameters of rooted product graphs were studied in [6].

Theorem 2.1. Let $G$ be a graph of order $n$ and let $H$ be any graph. If $S$ is a subset of vertices of $H$, then

$$
\gamma\left(G \square_{S} H\right) \geq n \gamma(H)-n|S|+|S| \gamma(G) .
$$

Proof. Let $V_{1}=\left\{u_{1}, u_{2}, \ldots, u_{n}\right\}$ and $V_{2}$ be the vertex sets of $G$ and $H$, respectively. Hence, we consider the set of vertices $S \subset V_{2}$. Let $D$ be a $\gamma\left(G \square_{S} H\right)$-set. For every $u_{i} \in V_{1}$, let $D_{i}=\left\{v \in V_{2}:\left(u_{i}, v\right) \in D\right\}$ and let $X_{i} \subset \overline{D_{i}}$ be the set of vertices of $H$ not dominated by $D_{i}$. Hence, we have that $D_{i} \cup X_{i}$ is a dominating set in $H$ and, as a consequence, $\gamma(H) \leq\left|D_{i}\right|+\left|X_{i}\right|$ for every is $i \in\{1, \ldots, n\}$. Hence,

$$
\begin{aligned}
|D| & =\sum_{i=1}^{n}\left|D_{i}\right| \\
& \geq \sum_{i=1}^{n}\left(\gamma(H)-\left|X_{i}\right|\right) \\
& =n \gamma(H)-\sum_{i=1}^{n}\left|X_{i}\right| .
\end{aligned}
$$

Thus, we have that

$$
\gamma\left(G \square_{S} H\right) \geq n \gamma(H)-\sum_{i=1}^{n}\left|X_{i}\right| .
$$

Notice that if $X_{j} \neq \emptyset$ for some $j \in\{1, \ldots, n\}$, then there exists a vertex $v \in V_{2}$ such that $\left(u_{j}, v\right)$ is dominated by a vertex $\left(u_{l}, v\right) \in D$ with $l \neq j$. Thus, $v \in S$. Hence, for every vertex $w \in S$ let $D_{w}=\left\{u_{i} \in V_{1}:\left(u_{i}, w\right) \in\left(\left\{u_{i}\right\} \times X_{i}\right)\right\}$. Notice that 
$\overline{D_{w}}$ is a dominating set in $G$. So, $\gamma(G) \leq\left|\overline{D_{w}}\right|=n-\left|D_{w}\right|$. Moreover, we have that $\sum_{i=1}^{n}\left|X_{i}\right|=\sum_{w \in S}\left|D_{w}\right|$.

Now, by using (2.1) we have the following.

$$
\begin{aligned}
\gamma\left(G \square_{S} H\right) & \geq n \gamma(H)-\sum_{i=1}^{n}\left|X_{i}\right| \\
& =n \gamma(H)-\sum_{w \in S}\left|D_{w}\right| \\
& \geq n \gamma(H)-\sum_{w \in S}(n-\gamma(G)) \\
& =n \gamma(H)-n|S|+|S| \gamma(G) .
\end{aligned}
$$

By taking a $\gamma(H)$-set $S$ in the above theorem we obtain a Vizing-like result for the domination number of the partial Cartesian product graph $G \square_{S} H$ with respect to $S$.

Corollary 2.2. Let $G$ and $H$ be any graphs. Then there exists a set $S$ of vertices of $H$ such that

$$
\gamma\left(G \square_{S} H\right) \geq \gamma(G) \gamma(H) .
$$

To see that the above bound is tight we consider the following. We say that a graph $G=(V, E)$ satisfies property $\mathcal{P}$ if it has a $\gamma(G)$-set $D$ such that $D=X \cup Y, X \cap Y=\emptyset$, $X$ dominates $V-Y$ and $Y$ dominates $V-X$. Let $\mathcal{F}$ be the family of all graphs satisfying property $\mathcal{P}$.

Proposition 2.3. Let $G$ be a graph with domination number half its order and let $H$ be a graph of the family $\mathcal{F}$. Then there exists a set $S$ of vertices of $H$ such that

$$
\gamma\left(G \square_{S} H\right)=\gamma(G) \gamma(H) .
$$

Proof. Since $H=(V, E)$ is a graph of the family $\mathcal{F}$, there exists a $\gamma(H)$-set $S$ such that $S=X \cup Y, X \cap Y=\emptyset, X$ dominates $V-Y$ and $Y$ dominates $V-X$. Also, as $G$ has domination number $\frac{n}{2}$, where $n$ is the order of $G$, we have that there exists a $\gamma(G)$-set $D$, such that $\gamma(G)=|D|=|\bar{D}|=\frac{n}{2}$. Notice that the set $(D \times X) \cup(\bar{D} \times Y)$ is a dominating set in $G \square_{S} H$ and, as a consequence,

$$
\gamma\left(G \square_{S} H\right) \leq|(D \times X) \cup(\bar{D} \times Y)|=|D||X|+|\bar{D}||Y|=\frac{n \gamma(H)}{2}=\gamma(G) \gamma(H) .
$$

The proof is finished by Corollary 2.2.

Since for every set $S$ of vertices of a graph $H$, the partial Cartesian product graph $G \square_{S} H$ is a subgraph of the Cartesian product graph $G \square H$, we have that $\gamma\left(G \square_{S} H\right) \geq$ $\gamma(G \square H)$. Hence, notice that if there exists a graph $G \square_{S} H$ such that $\gamma\left(G \square_{S} H\right)=$ $\gamma(G) \gamma(H)$ and $\gamma\left(G \square_{S} H\right)>\gamma(G \square H)$, then the Vizing's conjecture is not true. In this sense, the above results lead to the following question.

Question: Are there some graphs $G$ and $H$ such that some set $S$ of vertices of $H$ satisfies that $\gamma\left(G \square_{S} H\right)=\gamma(G) \gamma(H)$ and $\gamma\left(G \square_{S} H\right)>\gamma(G \square H)$ ? 
Next we study the independence domination number of the partial Cartesian product of graphs. A set $Y$ is an independent dominating set in a graph $G$, if $Y$ is a dominating set and there no edge between any two vertices of $Y$. The minimum cardinality of an independent dominating set of $G$ is the independence domination number of $G$ and it is denoted by $i(G)$. The following result from [6] will be useful to present our results.

Lemma 2.4. [6] Let $G=(V, E)$ be a graph. Then for every set of vertices $A \subset V$,

$$
i(G-A) \geq i(G)-|A| \text {. }
$$

Theorem 2.5. Let $G$ be a graph of order $n$ and let $H$ be any graph. If $S$ is a subset of vertices of $H$, then

$$
i\left(G \square_{S} H\right) \geq n(i(H)-|S|)+|S| i(G) .
$$

Proof. Let $V_{1}=\left\{u_{1}, u_{2}, \ldots, u_{n}\right\}$ and $V_{2}$ be the vertex sets of $G$ and $H$, respectively, and let $S \subset V_{2}$. Let $Y$ be an $i\left(G \square_{S} H\right)$-set. For every $u_{i} \in V_{1}$, we define the following subsets of vertices of $H$ :

- $Y_{i}=\left\{v \in V_{2}:\left(u_{i}, v\right) \in Y\right\}$,

- $S_{i} \subset S$ such that for every $v \in S_{i},\left(u_{i}, v\right)$ is independently dominated by some vertex $\left(u_{i}, v^{\prime}\right) \in Y$ with $v^{\prime} \in Y_{i}$,

- $R_{i} \subset S$ such that $R_{i}=S \cap Y_{i}$.

Thus, we have that for every $i \in\{1, \ldots, n\}, Y_{i}$ is an independent dominating set in $\left\langle V_{2}-\right.$ $\left.S+R_{i}+S_{i}\right\rangle$. So, by Lemma 2.4 we have that $\left|Y_{i}\right| \geq i\left(\left\langle V_{2}-S+R_{i}+S_{i}\right\rangle\right) \geq i(H)-$ $|S|+\left|R_{i}\right|+\left|S_{i}\right|$. Therefore, we obtain that

$$
|Y|=\sum_{i=1}^{n}\left|Y_{i}\right| \geq \sum_{i=1}^{n}\left(i(H)-|S|+\left|R_{i}\right|+\left|S_{i}\right|\right)=n(i(H)-|S|)+\sum_{i=1}^{n}\left(\left|R_{i}\right|+\left|S_{i}\right|\right) .
$$

Now, for every vertex $v \in S$ we define the following subset of vertices of $G$ :

- $A_{v}=\left\{u_{i} \in V_{1}:\left(u_{i}, v\right) \in Y\right\}$,

- $B_{v}=\left\{u_{i} \in V_{1}\right.$ : such that for every $\left.u_{i} \in B_{v},\left(u_{i}, v\right) \in S_{i}\right\}$, i.e., $\left(u_{i}, v\right)$ is independently dominated by some vertex $\left(u_{i}, v^{\prime}\right)$ where $v^{\prime} \in Y_{i}$.

Thus, we have that $A_{v}$ is an independent dominating set in $\left\langle V_{1}-B_{v}\right\rangle$ and, by Lemma 2.4, we have that $\left|A_{v}\right| \geq i\left(\left\langle V_{1}-B_{v}\right\rangle\right) \geq i(G)-\left|B_{v}\right|$. Moreover, notice that $\sum_{i=1}^{n}\left(\left|R_{i}\right|+\right.$ $\left.\left|S_{i}\right|\right)=\sum_{v \in S}\left(\left|A_{v}\right|+\left|B_{v}\right|\right)$. So, from Equation 2.2 we have the following.

$$
\begin{aligned}
|Y| & \geq n(i(H)-|S|)+\sum_{i=1}^{n}\left(\left|R_{i}\right|+\left|S_{i}\right|\right) \\
& =n(i(H)-|S|)+\sum_{v \in S}\left(\left|A_{v}\right|+\left|B_{v}\right|\right) \\
& \geq n(i(H)-|S|)+\sum_{v \in S}\left(i(G)-\left|B_{v}\right|+\left|B_{v}\right|\right) \\
& =n(i(H)-|S|)+|S| i(G) .
\end{aligned}
$$

Therefore, the result follows. 
Notice that, if $S$ is an $i(H)$-set, then the above result leads to item (i) of the next corollary . Moreover, if $S$ is formed by a single vertex, then the above result leads to a lower bound for the independence domination number of rooted product graphs, which was also obtained in [6].

Corollary 2.6. Let $G$ be a graph of order $n$ and let $H$ be any graph with vertex set $V$. Let $S \subset V$.

- If $S$ is an $i(H)$-set, then $i\left(G \square_{S} H\right) \geq i(G) i(H)$.

- If $S=\{v\}$, then $i\left(G \square_{S} H\right)=i\left(G \circ_{v} H\right) \geq n(i(H)-1)+i(G)$.

\subsection{Partial strong product of graphs}

Now, if $A \subset V_{1}$ and $B \subset V_{2}$, then the partial strong product of $G$ and $H$ with respect to $A$ and $B$ is the graph $G \otimes_{A, B} H=(V, E)$, where $V=V_{1} \times V_{2}$ and two vertices $\left(u_{i}, v_{j}\right)$ and $\left(u_{k}, v_{l}\right)$ are adjacent in $G \bigotimes_{A, B} H$ if and only if one of the following conditions is satisfied:

- $u_{i}=u_{k}$ and $v_{j} \sim v_{l}$,

- $u_{i} \sim u_{k}$ and $v_{j}=v_{l}$,

- $u_{i} \in A, v_{j} \in B, u_{i} \sim u_{k}$ and $v_{j} \sim v_{l}$,

- $u_{k} \in A, v_{l} \in B, u_{i} \sim u_{k}$ and $v_{j} \sim v_{l}$.

Notice that if $A=V_{1}$ and $B=V_{2}$, then $G \bigotimes_{A, B} H$ is the standard strong product graph $G \otimes H$. A more restrictive condition on the partial strong product of graphs could be the following one. If $A \subset V_{1}$ and $B \subset V_{2}$, then the restricted partial strong product of $G$ and $H$ with respect to $A$ and $B$ is the graph $G_{A} \bigotimes_{B} H=(V, E)$, where $V=V_{1} \times V_{2}$ and two vertices $\left(u_{i}, v_{j}\right)$ and $\left(u_{k}, v_{l}\right)$ are adjacent in $G_{A} \otimes_{B} H$ if and only if one of the following conditions is satisfied:

- $u_{i}=u_{k}$ and $v_{j} \sim v_{l}$,

- $u_{i} \sim u_{k}$ and $v_{j}=v_{l}$,

- $u_{i} \in A, u_{k} \notin A, v_{j} \in B, v_{l} \notin B, u_{i} \sim u_{k}$ and $v_{j} \sim v_{l}$,

- $u_{i} \notin A, u_{k} \in A, v_{j} \notin B, v_{l} \in B, u_{i} \sim u_{k}$ and $v_{j} \sim v_{l}$.

An example of a restricted partial strong product graph is shown in Figure 2.

Lemma 2.7. Let $G, H$ be any graphs and let $A$ (respectively $B)$ be a $\gamma(G)$-set (respectively $\gamma(H)$-set). Then $A \times B$ is a minimal dominating set in $G_{A} \bigotimes_{B} H$.

Proof. The proof follows from Lemma 1.1 and the procedure of construction of the restricted partial strong product of graphs.

We omit the proof of the following result, since it is straightforward.

Theorem 2.8. Let $G, H$ be any graphs. If $A$ and $B$ are dominating sets in $G$ and $H$, respectively, then

$$
\gamma\left(G \otimes_{A, B} H\right) \leq \gamma(G) \gamma(H) \text { and } \gamma\left(G_{A} \otimes_{B} H\right) \leq \gamma(G) \gamma(H) .
$$




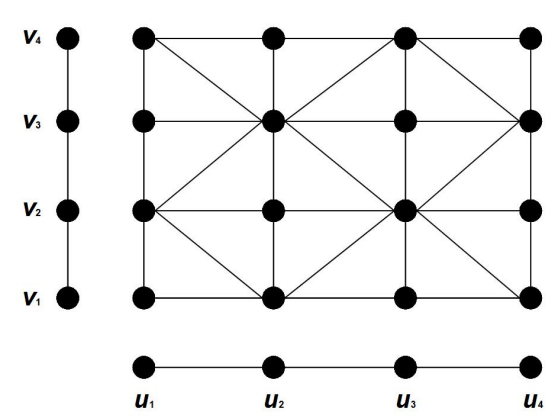

Figure 2: The restricted partial strong product graph $P_{4\left\{u_{1}, u_{3}\right\}} \bigotimes_{\left\{v_{2}, v_{4}\right\}} P_{4}$.

It is straightforward to observe that the partial Cartesian product graph $G \square_{S} H$ with respect to any set of vertices $S$ of $G$ is a subgraph of the restricted partial strong product graph $G_{A} \square_{B} H$ for any $\gamma(G)$-set $A$ and any $\gamma(H)$-set $B$. Thus, according to results of Theorem 2.1, Corollary 2.2 and Theorem 2.8 there would exist a spanning subgraph $F$ of the restricted partial strong product graph $G_{A} \square_{B} H$ satisfying that $\gamma(F)=\gamma(G) \gamma(H)$. In this sense, the question is: Is always the Cartesian product graph $G \square H$ a spanning subgraph of the graph $F$ ? If yes, then Vizing's conjecture is true.

\section{References}

[1] B. Brešar, P. Dorbec, W. Goddard, B. L. Hartnell, M. A. Henning, S. Klavžar and D. F. Rall, Vizing's conjecture: a survey and recent results, Journal of Graph Theory 69 (2012), 46-76.

[2] C. D. Godsil and B. D. McKay, A new graph product and its spectrum, Bulletin of the Australasian Mathematical Society 18 (1) (1978), 21-28.

[3] B. Hartnell and D. F. Rall, Domination in Cartesian products: Vizing's conjecture. In Domination in graphs, volume 209 of Monography and Textbooks in Pure and Applied Mathematics, pages 163-189, Marcel Dekker, New York, 1998.

[4] T. W. Haynes, S. T. Hedetniemi and P. J. Slater, Fundamentals of Domination in Graphs, Marcel Dekker, Inc. New York, 1998.

[5] R. Hammack, W. Imrich and S. Klavžar, Handbook of product graphs, volume 66 of Discrete Mathematics and its Applications, CRC Press, 2011.

[6] D. Kuziak, M. Lemańska and I. G. Yero, Domination related parameters in rooted product graphs, Bulletin of the Malaysian Mathematical Sciences Society (2013), in press.

[7] V. G. Vizing, The Cartesian product of graphs, Vyčisl. Sistemy 9 (1963), 30-43. 